\title{
Comparison of Innovator vs. Biosimilar Ranibizumab in Treating Diabetic Macular Edema: A Multicenter Retrospective Study
}

\author{
Debdulal Chakraborty (D) - Sabyasachi Sengupta · Soumen Mondal • \\ Subhendu Boral · Arnab Das · Tushar Kanti Sinha · Ranabir Bhattacharya • \\ Ritobroto Maitra
}

Received: December 18, 2021 / Accepted: January 11, 2022 / Published online: January 24, 2022

(C) The Author(s) 2022

\section{ABSTRACT}

Introduction: To compare the efficacy of innovator ranibizumab (iRBZ-Accentrix, Novartis, India) vs. biosimilar ranibizumab (bRBZ, Razumab-Intas, India) in eyes with diabetic macular edema (DME) in an Indian population.

Methods: Data of patients with DME who underwent at least three injections of iRBZ or bRBZ and had a minimum of 6 months followup were obtained from an electronic database. Choice of injection depended upon the patient. Pro re nata (PRN) protocol from baseline was used with reinjections advised if the central macular thickness (CMT) was at least $300 \mu \mathrm{m}$

D. Chakraborty $(\bowtie) \cdot S$. Mondal .

S. Boral - A. Das - T. K. Sinha - R. Bhattacharya Department of Vitreo-Retinal Services, Disha Eye Hospitals, 88 Ghosh Para Road Barrackpore, Kolkata, West Bengal 700120, India e-mail: devdc@rediffmail.com

S. Mondal

e-mail: hisoumenm@yahoo.co.in

S. Boral

e-mail: drsubhendu@yahoo.co.uk

A. Das

e-mail: drarnabdas_aiims@rediffmail.com and best corrected visual acuity (BCVA) was 20/40 or worse. Primary outcome measure was comparison of change in BCVA at 6 months between iRBZ and bRBZ.

Results: We included 264 eyes in the iRBZ group and 69 eyes in bRBZ group, which were comparable for baseline characteristics. Mean BCVA improved from $0.64 \pm 0.39 \log$ MAR to $0.47 \pm 0.31 \log$ MAR $(p<0.001)$ in the iRBZ group and from $0.71 \pm 0.42 \log \mathrm{MAR}$ to $0.50 \pm 0.29 \log \mathrm{MAR}$ in the bRBZ group $(p<0.001)$ at 6 months. There were no differences in BCVA between the two groups ( $p>0.05$ for all time points). The CMT reduction in the iRBZ group $(120 \pm 196 \mu \mathrm{m})$ was comparable to that in the bRBZ group at

\author{
T. K. Sinha \\ e-mail: drtusharsinha@gmail.com \\ R. Bhattacharya \\ e-mail: ranabirbh@gmail.com
}

\author{
S. Sengupta \\ Future Vision Eye Care and Research Centre, \\ Mumbai, India \\ e-mail: drsunny1980@gmail.com \\ R. Maitra \\ Radical Health-Tech Private Limited, New Delhi, \\ India \\ e-mail: rito@radicalhealth.in
}


6 months $(105 \pm 187 \mu \mathrm{m})(p=0.69)$. There was no difference in the mean number of injections taken $(3.81 \pm 1.2$ in $\mathrm{iRBZ}$ vs. $3.55 \pm 1.2$ in bRBZ) $(p>0.05)$ between groups. Vision at baseline was the only factor associated with vision at last follow-up after adjusting for CMT at baseline, type of injection, and number of injections.

Conclusions: Biosimilar RBZ is similar to innovator RBZ in improving vision and reducing CMT in eyes with DME in the short term.

Keywords: Anti-VEGF;

Ranibizumab;

Biosimilar ranibizumab; Diabetic macular edema

\section{Key Summary Points}

\section{Why carry out this study?}

Biosimilars of anti-vascular endothelial growth factor (anti-VEGF) drugs are an appealing therapeutic alternative to the parent biologic since they have the potential to reduce the cumulative cost of treatment in various chorioretinal disorders. Although the Indian drug regulator-approved ranibizumab biosimilar Razumab (Intas

Pharmaceuticals, Ahmedabad, India) has demonstrated good efficacy and safety based on phase III studies, the limited number of patients treated in a controlled environment may not truly reflect the delivery settings and the population diversities in real-world scenarios.

\section{What was learned from the study?}

In a real-world setting, biosimilar ranibizumab is similar to innovator ranibizumab in improving vision and reducing central macular thickness (CMT) in eyes with diabetic macular edema (DME) in the short term. Intravitreal biosimilar ranibizumab has an acceptable ocular and systemic safety profile in patients with diabetic macular edema.

\section{INTRODUCTION}

Diabetic macular edema (DME) is one of the leading causes of visual impairment globally and the increasing prevalence of diabetes means that there will be an exponential rise in the number of patients with DME $[1,2]$. The treatment of DME has seen paradigm shifts in the last decade due to the use of intravitreal antivascular endothelial growth factor (anti-VEGF) agents [3]. Various trials under the Diabetic Retinopathy Clinical Research Network have clearly shown the efficacy of ranibizumab (Lucentis, Genentech, South San Francisco, CA, USA) and aflibercept (Eylea, Regeneron Pharmaceuticals, Inc, Tarrytown, New York, and Bayer HealthCare, Berlin Germany) in improving outcomes in eyes with DME [4]. However, the need for repeated treatments on a monthly basis leads to an increasing burden of treatment and affordability becomes an issue when patients are paying out of pocket, as happens in most of the developing world. Even though ranibizumab is available at a substantially reduced cost in India (Accentrix, Novartis India Ltd, Mumbai, India), patients still find it difficult to take repeated injections $[5,6]$.

In view of the increasing demand for antiVEGF injections and the cost-prohibitive nature of treatment, retina physicians resort to the use of cheaper treatment options. Bevacizumab (Avastin, Genentech, South San Francisco, CA and Roche, Basel, Switzerland) is the commonest agent used in an off-label form globally and in a recent survey by the American Society of Retina Specialists, it was the commonest drug used in practice in the majority of the western world [7]. However, its use can be associated with cluster endophthalmitis as seen in India recently [8]. Biosimilars are very similar agents to approved reference biological products, with no clinically meaningful differences in terms of efficacy, safety, and immunogenicity [9]. These are generally available at lower costs compared to the innovator molecules and are another way to reduce cost of treatment. In India, a ranibizumab biosimilar (Razumab, Intas pharmaceuticals, Ahmedabad, India) was approved for intravitreal use in 2015 and has been 
increasingly adopted by retina physicians [10]. It has shown good efficacy for most retinal disorders in limited studies and more than 100,000 injections have already been used in India alone [11]. However, to the best of our knowledge, there are no comparative studies showing the efficacy of this biosimilar (bRBZ) with the innovator ranibizumab (iRBZ) molecule with up to 1-year follow-up.

Ours is a network of tertiary eye care centers and teaching hospitals in eastern India and we cater to more than a million patients annually. We have been increasingly adopting the bRBZ in our institution over the past 4 years as confidence in this molecule grows; currently, more than half of all our patients receive bRBZ. As an institutional protocol and in view of financial constraints faced by our patients, we use a pro re nata (PRN) regimen right from baseline in eyes with DME. In this study, we present a retrospective analysis of outcomes of bRBZ compared to iRBZ in eyes with DME in an Indian population.

\section{METHODS}

This was a multicentric retrospective comparative study performed at a group of eye hospitals in eastern India. The study was conducted in accordance with the Declaration of Helsinki and was approved by the Master Ethical Committee of Disha Eye Hospitals in Kolkata and by the institutional review board at each center (Regn Number ECR/846/Inst/WB/2016/RR-19: EC-CT2020-138). All patients gave informed consent before undergoing intravitreal injections. Data were collected from four centers within our hospital network and patients were treated by 10 fellowship trained retina specialists.

All patients over 18 years of age with a diagnosis of diabetic macular edema (ICD-9 coding) having a baseline visual acuity of $20 / 40$ Snellen's equivalent ( 0.3 logarithm of minimum angle of resolution, logMAR) or worse, who underwent intravitreal iRBZ or bRBZ for the first time across our hospital network between January 2015 and March 2020 were identified from a centralized electronic medical records system. All relevant data from those with a monthly follow-up of 6 months minimum and taken at least three injections during the first 6 months were entered into a datasheet. Only patients with a baseline HbA1c of 8.0 or less were included. Lastly, we included eyes matched for similar follow-up durations between the two injection groups for comparative analysis using statistical methods. A flowchart of the selection process is shown in Fig. 1. All patients with any diagnosis other than DME, significant pathology interfering with vision such as visually significant cataract, glaucoma, or any other pathologies causing macular edema and vitreoretinal interface disorders were excluded. Similarly, all eyes with any missing data such as best corrected visual acuity (BCVA) or macular thickness at any time point were also excluded. We also excluded eyes

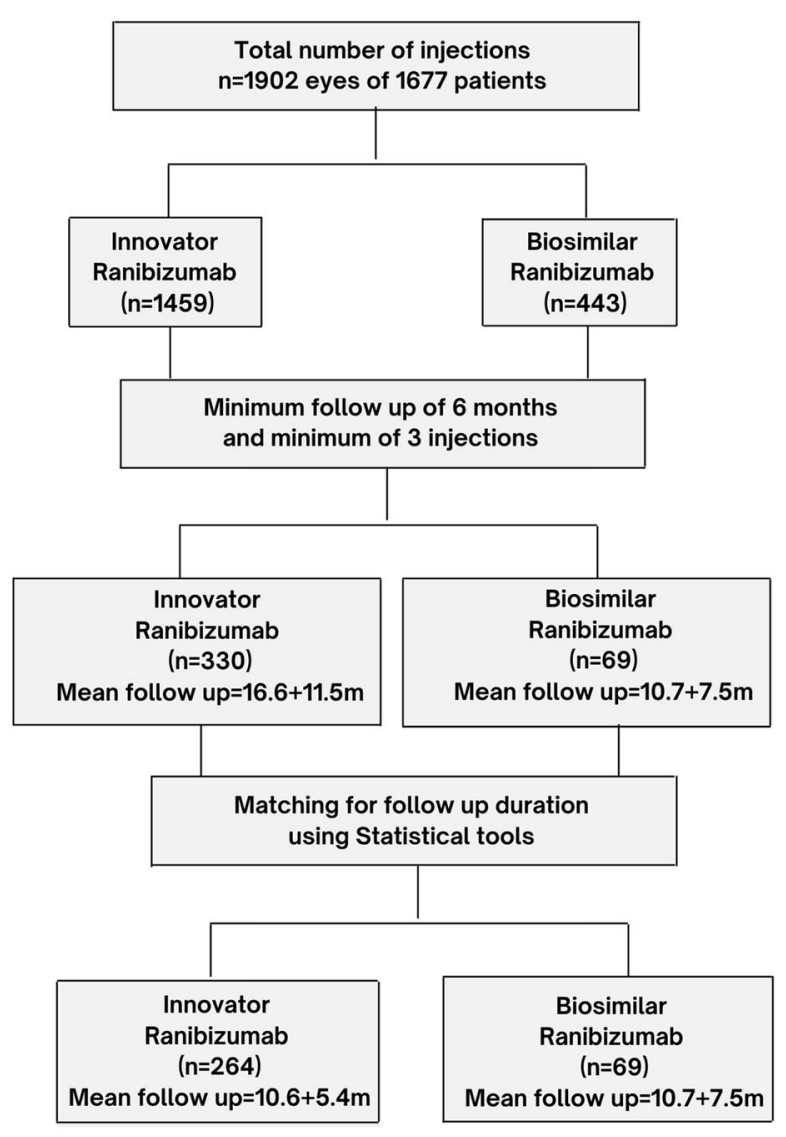

Fig. 1 Flowchart showing methods of inclusion of patients for the comparative study 
with DME and coexistent proliferative diabetic retinopathy, macular ischemia, previous intravitreal injections or retinal photocoagulation, or any previous vitreous surgery.

All patient data including demographics, BCVA, intraocular pressure, slit lamp examination to assess the lens status, and fundus evaluation was recorded. This was followed by optical coherence tomography (OCT)-based assessment of the central macular thickness (CMT). Measurements were obtained using two different spectral domain OCT machines, namely Optovue OCT (Freemont, CA, USA) and at two centers and Spectralis OCT (Heidelberg, Germany) at two other centers. Automated CMT display was recorded at each visit from scans with a minimum image quality of $50 \%$. The same machine was used for measurements at all time points for each patient; also the patients were examined at the same center on each visit. Fundus fluorescein angiography findings were recorded from case files when available.

Patients with DME were offered all information about the two injections, i.e., iRBZ (Accentrix, Novartis, India) and bRBZ (Razumab, Intas Pharmaceuticals, India), and informed about the potential need for repeated injections. The injection was based on the patient's choice and drug affordability. As per our institutional protocol, we adopted a PRN retreatment regimen from baseline with monthly re-evaluation for the first 6 months. Reinjections were advised if the CMT was at least $300 \mu \mathrm{m}$ and BCVA was 20/40 or worse at any time point during the first 6 months. The number of reinjections taken at each monthly time point was noted. Follow-up times were prolonged after 6 months, with increments of 2 weeks at a time, if reinjection was not required at the 6th month time point.

Comparison of change in visual acuity at 6 months time point $(\triangle \mathrm{BCVA}=$ baseline BCVA - 6th month BCVA) between iRBZ and bRBZ was the primary outcome measure. Change in CMT, number of reinjections, and three-line gainers and losers were the secondary outcome measures.

All continuous variables were expressed as means with standard deviation or median with interquartile range (IQR) and group differences between continuous variables were analyzed using Student's $t$ test or Wilcoxon's ranksum test for non-parametric distributions. Categorical variables were expressed as proportions ( $n$, $\%)$ and group differences were analyzed using the chi-square or the Fischer's exact test. Comparisons between parameters at baseline and at various time points during the study were done using the analysis of variance with Bonferroni adjustments.

Selection of matched eyes with respect to follow-up duration between innovator and biosimilar ranibizumab was done using the rangejoin command in STATA with a follow-up range for the innovator set within 1 month of the biosimilar. In view of repeated measures, a random effects linear regression model was used to determine factors influencing change in BCVA defined as BCVA at baseline minus BCVA at last follow-up.

All data was maintained in Microsoft Excel and analyzed using STATA 12.1 I/c (Stata Corp, Fort Worth, Texas, USA) and all $p$ values less than 0.05 were considered statistically significant.

\section{RESULTS}

Out of a possible 1902 eyes of 1677 unique patients that received either the iRBZ or bRBZ during the study period, inclusion criteria were satisfied by 403 eyes (21\%) (Fig. 1). Following the matching process done for similar follow-up periods, we included 333 eyes of 303 patients in this analysis. Of eyes included, 264 received the iRBZ while the remaining 69 received the bRBZ. There were no differences in baseline characteristics, follow-up duration, and mean number of injections (Table 1) in iRBZ and bRBZ groups.

The overall mean baseline BCVA improved from $0.66 \pm 0.39 \log$ MAR to $0.49 \pm 0.32$ at 3 months $(p<0.001)$ and then stabilized at 6 months $(0.41 \pm 0.31 \log$ MAR, $p=0.18$ compared to 3 months) and 1 year $(0.39 \pm 0.31 \log$ MAR, $p=0.65$ compared to 6 months) follow-up. Comparison of BCVA between the two groups (Fig. 2a) showed no differences at all time points (Table 2). There 
Table 1 Comparison between demographics, baseline vision, CMT, follow-up duration, and mean number of injections between innovator and biosimilar ranibizumab

\begin{tabular}{llll}
\hline Variable & Innovator RBZ $(\boldsymbol{n}=\mathbf{2 6 4})$ & Biosimilar RBZ $(\boldsymbol{n}=\mathbf{6 9})$ & $\boldsymbol{p}$ value \\
\hline Age (years) & $57.5 \pm 3.8$ & $58.6 \pm 4.2$ & 0.67 \\
Gender $(\%$ men) & $165(62 \%)$ & $42(61 \%)$ & 0.77 \\
Eye (\% right eye) & $128(48 \%)$ & $34(49 \%)$ & 0.91 \\
BCVA at baseline (logMAR) & $0.64 \pm 0.39$ & $0.71 \pm 0.42$ & 0.18 \\
CMT at baseline ( $\mu$ m) & $457 \pm 172$ & $434 \pm 153$ & 0.40 \\
Follow-up duration (months) & $10.6 \pm 5.5$ & $10.7 \pm 7.5$ & 0.31 \\
Mean number of injections & $3.81 \pm 1.2$ & $3.55 \pm 1.2$ & 0.33 \\
\hline
\end{tabular}

$R B Z$ ranibizumab, $B C V A$ best corrected visual acuity, $C M T$ central macular thickness
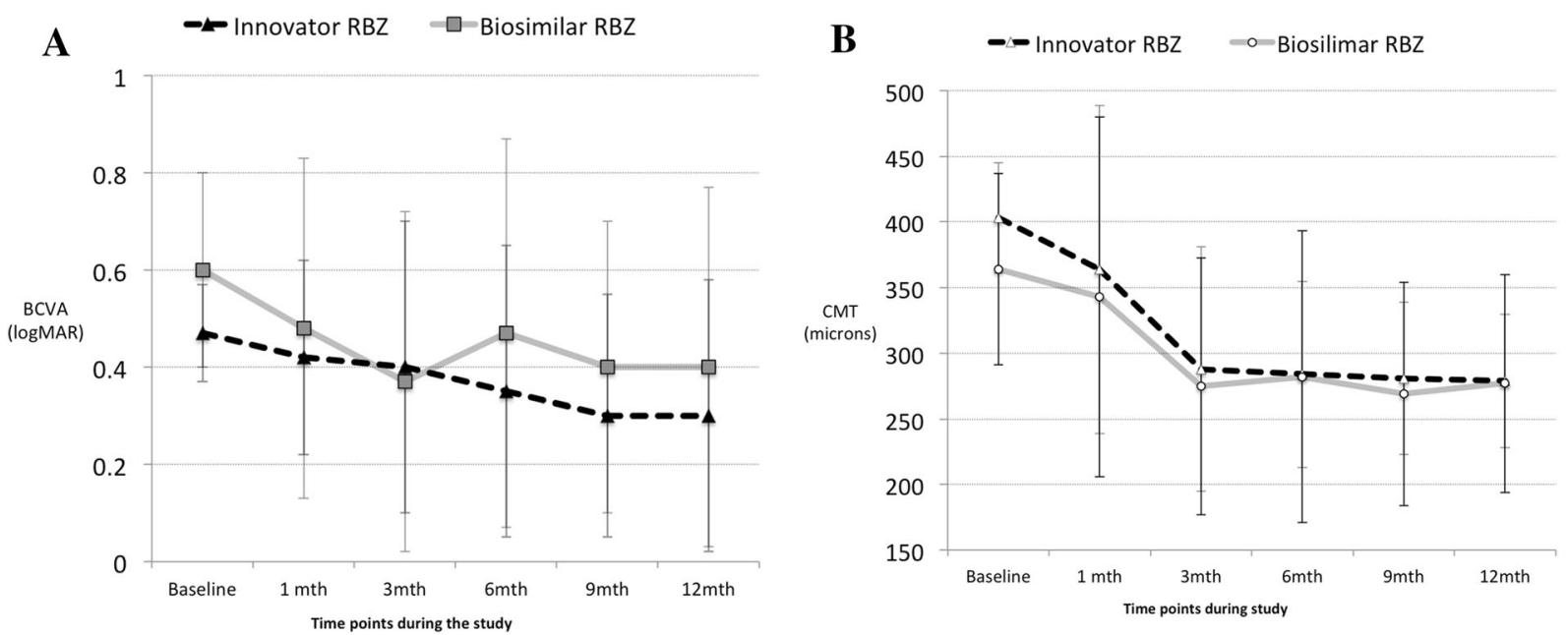

Fig. 2 Comparison of the a median best corrected visual acuity (with the $95 \%$ confidence intervals) and $\mathbf{b}$ median central macular thickness (with the $95 \%$ confidence intervals) between innovator and biosimilar ranibizumab groups

was a mean visual improvement of $0.24 \pm 0.39 \log$ MAR units overall at the last follow-up. A three-line gain in BCVA was seen in 146 eyes (44\%) while a three-line loss was seen in 19 eyes $(6 \%)$.

Similarly, the overall mean CMT reduced from $452 \pm 169 \mu \mathrm{m}$ at baseline to $360 \pm 145 \mu \mathrm{m} \quad$ at 1 month $\quad(p=0.03)$, $325 \pm 137 \mu \mathrm{m}$ at 3 months $(p=0.03$ compared to 1 month), and then remained stable at $342 \pm 161 \mu \mathrm{m}$ at 6 months $(p=0.31$ compared to 3 months $)$ and $336 \pm 155 \mu \mathrm{m} \quad(p=0.73$ compared to 6 months) at 1-year follow-up. Comparison of CMT between the two groups (Fig. 2b) showed no differences at all time points (Table 2). Overall, the CMT reduced by $117 \pm 196 \mu \mathrm{m}$ at the last follow-up, with no statistically significant differences between groups (Table 2). There was no difference in the mean number of injections taken $(3.81 \pm 1.2$ in iRBZ vs. $3.55 \pm 1.2$ in bRBZ) $(p>0.05)$ between groups. A total of 299 (90\%) eyes required a loading dose $[n=240$ in iRBZ (91\%) and $n=59$ $(86 \%)$ in bRBZ group] but this was taken by only 
Table 2 Comparison of vision and macular thickness at different time points between the innovator and biosimilar ranibizumab

\begin{tabular}{|c|c|c|c|}
\hline Variable & Innovator RBZ $(n=264)$ & Biosimilar RBZ $(n=69)$ & $p$ value \\
\hline \multicolumn{4}{|c|}{ BCVA comparisons (logMAR) } \\
\hline Baseline & $0.64 \pm 0.39$ & $0.71 \pm 0.42$ & 0.18 \\
\hline 1 month & $0.48 \pm 0.29^{* *}$ & $0.55 \pm 0.30^{* *}$ & 0.32 \\
\hline 3 months & $0.49 \pm 0.39$ & $0.49 \pm 0.25$ & 0.72 \\
\hline 6 months & $0.47 \pm 0.31$ & $0.50 \pm 0.29$ & 0.61 \\
\hline 9 months & $0.37 \pm 0.32(n=134)$ & $0.47 \pm 0.27(n=41)$ & 0.17 \\
\hline 12 months & $0.38 \pm 0.32(n=123)$ & $0.44 \pm 0.26(n=35)$ & 0.25 \\
\hline Final visual acuity* & $0.40 \pm 0.36$ & $0.44 \pm 0.26$ & 0.39 \\
\hline BCVA & $0.24 \pm 0.40$ & $0.26 \pm 0.34$ & 0.71 \\
\hline$\% 3$ line loss & $18(7 \%)$ & $1(1.5 \%)$ & 0.14 \\
\hline$\% 3$ line gain & $114(43 \%)$ & $32(46 \%)$ & 0.63 \\
\hline \multicolumn{4}{|c|}{ CMT comparisons $(\mu \mathrm{m})$} \\
\hline Baseline & $457 \pm 172$ & $434 \pm 153$ & 0.40 \\
\hline 1 month & $364 \pm 152^{* *}$ & $343 \pm 126^{* *}$ & 0.88 \\
\hline 3 months & $334 \pm 147^{* *}$ & $306 \pm 97^{* *}$ & 0.51 \\
\hline 6 months & $346 \pm 162$ & $326 \pm 159$ & 0.62 \\
\hline 9 months & $342 \pm 161(n=134)$ & $316 \pm 150(n=41)$ & 0.45 \\
\hline 12 months & $341 \pm 161(n=134)$ & $318 \pm 150(n=41)$ & 0.58 \\
\hline CMT final* & $337 \pm 160$ & $328 \pm 137$ & 0.83 \\
\hline CMT & $120 \pm 196$ & $105 \pm 187$ & 0.69 \\
\hline
\end{tabular}

* Any time period

${ }^{* *} p<0.05$ compared to previous time point

$R B Z$ ranibizumab, $C M T$ central macular thickness

139 (42\%). None of the eyes included in this study experienced any adverse reactions such as intraocular inflammation or endophthalmitis, and none of the patients suffered any drug-related systemic adverse events such as thromboembolism, myocardial infarction, or stroke during the follow-up period.

\section{DISCUSSION}

We found no differences in the visual and anatomical outcomes between treatment-naïve eyes with DME that received the innovator vs. the biosimilar ranibizumab. Overall, visual improvement or stabilization was seen in the majority of patients with very few experiencing three or more lines of visual loss in the short term. Visual recovery was seen from 1 month onwards in both groups and was sustained over time despite less than $50 \%$ adhering to the advised treatment regimens. The vision was consistently better by half a line in the iRBZ group right from baseline but differences were not significant at any of the time points. 
Table 3 Comparison of number of injections in the innovator and biosimilar groups

\begin{tabular}{lclc}
\hline Variable & Innovator RBZ $(\boldsymbol{n}=\mathbf{2 6 4})$ & Biosimilar RBZ $(\boldsymbol{n}=\mathbf{6 9})$ & $\boldsymbol{p}$ value \\
\hline 3 injections & $141(53 \%)$ & $50(72.5 \%)$ & 0.13 \\
4 injections & $74(28 \%)$ & $11(16 \%)$ & \\
5 injections & $23(9 \%)$ & $3(4 \%)$ & \\
6 injections & $14(5 \%)$ & $2(3 \%)$ & \\
7 injections & $7(3 \%)$ & $1(1.5 \%)$ & \\
8 injections & $4(1.5 \%)$ & $1(1.5 \%)$ & \\
9 injections & $1(0.5 \%)$ & $1(1.5 \%)$ & 0.004 \\
\hline eyes receiving 3 loading doses & $118(45 \%)$ & $21(30 \%)$ & \\
\hline
\end{tabular}

$R B Z$ ranibizumab, $C M T$ central macular thickness

Macular edema also decreased over time and equally well in both groups.

The eyes in the iRBZ group received slightly more injections during the follow-ups (Table 3), but these differences were not statistically significant. However, a significantly greater proportion of eyes in the iRBZ group received a loading dose of three monthly injections in the first 3 months compared to bRBZ. On the basis of retreatment criteria, though 299 (90\%) eyes required a loading dose only 139 (42\%) actually went on to take the loading dose. In real-life scenarios, patients actually not being able to take loading dose of three injections is a reality and is depicted in our study also. A multivariable linear regression analysis using a random effects modeling showed that lower BCVA at baseline was the only factor associated with significantly more gain in vision at last followup (every one-line worsening in baseline BCVA was associated with $0.06 \log$ MAR greater gain in vision, $\quad 95 \% \quad \mathrm{CI}=0.053-0.071 \log \mathrm{MAR}$, $p<0.001)$ after adjusting for CMT at baseline, type of injection, and number of injections. A correlation between BCVA at baseline and last follow-up is shown in Fig. 3.

The availability of biosimilars has added to our armamentarium of anti-VEGF drugs and enabled us to offer retreatments at reduced costs $[5,9]$. However, lack of comparative studies with a significant period of follow-up with the innovator molecule has led to skepticism

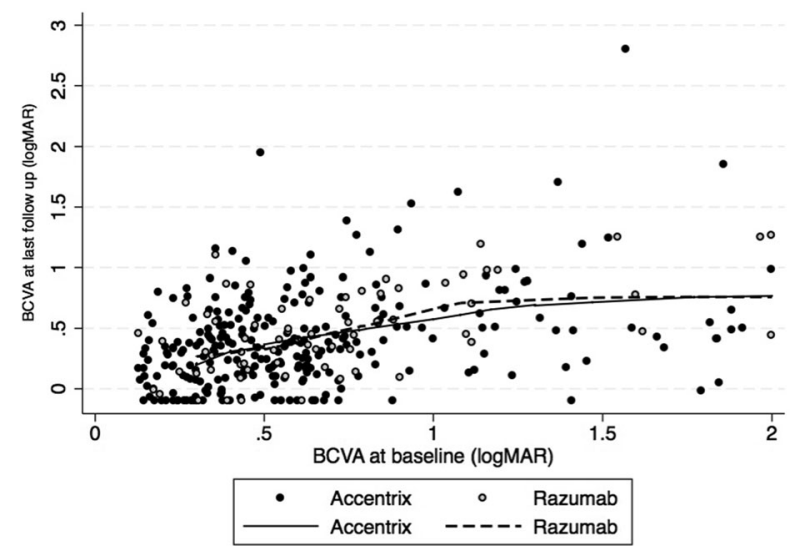

Fig. 3 Scatter plot with a locally weighted smoothening curve showing the correlation between best corrected visual acuity at baseline and last follow-up

amongst physicians and has prevented widespread adoption. Our results are encouraging and should alleviate concerns about efficacy of the biosimilar for management of DME. Previous non-comparative studies using the same biosimilar have also shown good results in eyes with DME. In the recently published CESAR study [12], Verma et al. report excellent visual outcomes from 57 treatment-naïve DME eyes (median of $0.5 \log$ MAR to $0.2 \log$ MAR at 3 months) that received bRBZ as a PRN protocol from baseline. They also report improvement in CMT from $436.70 \pm 174.33 \mu \mathrm{m}$ at baseline to $275.04 \pm 120.09 \mu \mathrm{m}$ at 3 months. In another retrospective study on 44 Indian eyes with 
DME, Sameera et al. showed a one-line visual improvement at 1 month using bRBZ for both treatment-naïve $(n=18)$ and pretreated eyes [13]. Similarly, the macular thickness also reduced from $366.8 \pm 93.9 \mu \mathrm{m}$ to $311.6 \pm 83.2 \mu \mathrm{m} \quad(p<0.001)$, similar to our results. Since Sameera et al.'s study was preliminary, they performed electroretinography for all patients, which did not show any appreciable changes at 1 month [13]. However, in their study, further follow-up was lacking, making it difficult to interpret the efficacy of the drug. In a more recent study, Gopal et al. retrospectively studied 120 eyes with DME and again showed similar visual improvement from 0.59 to $0.47 \log$ MAR units and CMT reduction from $393 \mu \mathrm{m}$ to $321 \mu \mathrm{m}$ at 1 month using bRBZ, similar to our results [14]. However, follow-up data beyond 1 month was again lacking. In a large retrospective multicentric study (REENACT study) pooling data from 17 centers across India, Sharma et al. reported treatment outcomes using bRBZ in 341 eyes with various retinal conditions of which 103 eyes had DME [15]. Though the authors do not provide outcomes for individual disease pathologies, they show significant cumulative visual improvement in the majority of eyes. Our study is the first with up to 12 months follow-up comparing iRBZ and bRBZ in eyes with DME and shows sustained visual gains maintained at final follow-up, despite PRN treatment protocols from baseline.

In resource-poor settings where payments are predominantly out of pocket, most patients find it hard to adopt monthly loading doses for the first 3 months. Additionally, patients' lower educational status also means that they fail to perceive the benefits of treatment and are often unsatisfied with visual outcomes [6]. These factors mean that the majority of physicians in the developing world, including India, are forced to adopt a PRN treatment protocol from baseline, as evidenced by a lot of studies published from the region $[10,12,16]$. Previous studies from the west have also shown that about a third of the patients can do well even without the loading dose, provided a close follow-up can be maintained and reinjections given at the first instance of requirement based on established retreatment criteria [17]. Our results and others show that the majority of eyes maintain vision and almost half experience good visual gain adopting this strategy. Given the financial and other constraints, a PRN strategy from baseline with strict follow-up and timely reinjections can be adopted in resource-poor settings to minimize the number of injections without losing treatment benefit in eyes with DME. On the basis of our results, we also believe that patients with DME adopting the PRN from baseline should be counseled about the need for a minimum of four injections in the first year in addition to the burden of monthly follow-up visits and OCT scans for at least the first 6 months.

The merits of our study are the relatively large sample size and comparisons between iRBZ and bRBZ, with 12 months follow-up, that have not been reported in the past, to the best of our knowledge. However, strict inclusion criteria meant that many patients who did not take injections as advised and did not undergo follow-up were excluded. Additionally, having different OCT machines across centers may introduce an element of error in the overall CMT results, though using the same machine at all visits may minimize estimates.

\section{CONCLUSION}

The bRBZ performed as well as the iRBZ in terms of visual outcomes and reductions in macular thickness over 1-year follow-up. Adopting a PRN regimen from baseline to treat DME may be a viable strategy in resource-poor settings like ours but overall results may be still inferior to more frequent injections. On the basis of our results, bRBZ can be adopted by more vitreoretina specialists so that the cost of treatment can be brought down further without compromising on visual and anatomic results.

\section{ACKNOWLEDGEMENTS}

We thank the participants of the study and Dr Debasish Bhattacharya, Chairman of Disha Eye 
Hospitals and the entire Vitreo-Retina Department of Disha Eye Hospitals.

Funding. No funding or sponsorship was received for this study or publication of this article. The Rapid Service Fee was funded by the authors.

Editorial Assistance. Editorial assistance was provided by Sengupta's Research Academy, Mumbai, India.

Authorship. All named authors meet the International Committee of Medical Journal Editors (ICMJE) criteria for authorship for this article, take responsibility for the integrity of the work as a whole, and have given their approval for this version to be published.

Author Contributions. All authors made substantial contributions to the conception, design of the study and analysis, and interpretation of data. Debdulal Chakraborty, Sabyasachi Sengupta, Soumen Mondal, Subhendu Boral, Arnab Das, Tushar Kanti Sinha all contributed significantly to the conception, design, analysis, interpretation of the data and review of the draft. Ranabir Bhattacharya and Ritobroto Maitra collected the data, coded and analyzed data.

Disclosures. Debdulal Chakraborty, Sabyasachi Sengupta, Soumen Mondal, Subhendu Boral, Arnab Das, Tushar Kanti Sinha, Ranabir Bhattacharya and Ritobroto Maitra all have nothing to disclose.

Compliance with Ethics Guidelines. This study was performed in line with the principles of the Declaration of Helsinki. Approval was granted by the Ethics Committee of Disha Eye Hospitals in Kolkata (Regn Number ECR/846/ Inst/WB/2016/RR-19: EC-CT-2020-138).

Data Availability. The datasets generated during and/or analyzed during the current study are available from the corresponding author on reasonable request.
Open Access. This article is licensed under a Creative Commons Attribution-NonCommercial 4.0 International License, which permits any non-commercial use, sharing, adaptation, distribution and reproduction in any medium or format, as long as you give appropriate credit to the original author(s) and the source, provide a link to the Creative Commons licence, and indicate if changes were made. The images or other third party material in this article are included in the article's Creative Commons licence, unless indicated otherwise in a credit line to the material. If material is not included in the article's Creative Commons licence and your intended use is not permitted by statutory regulation or exceeds the permitted use, you will need to obtain permission directly from the copyright holder. To view a copy of this licence, visit http:// creativecommons.org/licenses/by-nc/4.0/.

\section{REFERENCES}

1. Adelson JD, Bourne RRA, Briant PS, et al. Causes of blindness and vision impairment in 2020 and trends over 30 years, and prevalence of avoidable blindness in relation to VISION 2020: the Right to Sight: an analysis for the Global Burden of Disease Study. Lancet Glob Health. 2021;9(2):e144-60.

2. Lee R, Wong TY, Sabanayagam C. Epidemiology of diabetic retinopathy, diabetic macular edema and related vision loss. Eye Vis (Lond). 2015;30(2):17.

3. Mansour SE, Browning DJ, Wong K, Flynn HW Jr, Bhavsar AR. The evolving treatment of diabetic retinopathy. Clin Ophthalmol. 2020;14:653-78.

4. Cai S, Bressler NM. Aflibercept, bevacizumab or ranibizumab for diabetic macular oedema: recent clinically relevant findings from DRCR.net Protocol T. Curr Opin Ophthalmol. 2017;28(6):636-43.

5. Sengupta S. Current perspectives on use of antivascular endothelial growth factor agents for retinal disorders. Indian J Ophthalmol. 2021;69(2):209.

6. Kelkar A, Webers C, Shetty R, et al. Factors affecting compliance to intravitreal anti-vascular endothelial growth factor therapy in Indian patients with retinal vein occlusion, age-related macular degeneration, and diabetic macular edema. Indian J Ophthalmol. 2020;68(10):2143. 
7. American Society of Retina Specialists. ASRS Preferences and Trends (PAT) membership survey 2018. ASRS. https://www.asrs.org/asrs-community/patsurvey. Accessed 6 Jan 2021.

8. Stewart MW, Narayanan R, Gupta V, Rosenfeld PJ, Martin DF, Chakravarthy U. Counterfeit avastin in India: punish the criminals, not the patients. Am J Ophthalmol. 2016;170:228-31.

9. Sharma A, Reddy P, Kuppermann BD, Bandello F, Loewenstein A. Biosimilars in ophthalmology: "is there a big change on the horizon?" Clin Ophthalmol. 2018;12:2137-43.

10. Sheth JU, Stewart MW, Khatri M, et al. Changing trends in the use of anti-vascular endothelial growth factor (anti-VEGF) biosimilars: insights from the Vitreoretinal Society of India Biosimilars of Anti-VEGF Survey. Indian J Ophthalmol. $2021 ; 69(2): 352$.

11. Chakraborty D, Stewart MW, Sheth JU, et al. Realworld safety outcomes of intravitreal ranibizumab biosimilar (Razumab) therapy for chorioretinal diseases. Ophthalmol Ther. 2021;10:337-48. https:// doi.org/10.1007/s40123-021-00345-2.

12. Verma L, Thulasidas M, Purohit A, Gupta A, Narula R, Talwar D. Clinical efficacy and safety of Razumab® (CESAR) study: our experience with the world's first biosimilar ranibizumab. Indian J Ophthalmol. 2021;69:347-51.
13. Sameera VV, Apoorva AG, Joshi S, Guruprasad AS. Safety and efficacy of Razumab - the new biosimilar in India: our experience. Kerala J Ophthalmol. 2016;28:180-5.

14. Gopal S, Kasturirangan S, Madhivanan N, Henry H, Nivean PD, Shekharan S. Clinical effectiveness and safety of Razumab (a biosimilar of ranibizumab). TNOA J Ophthalm Sci Res. 2020;58:154-8.

15. Sharma S, RE-ENACT 2 Study Investigators Group, Khan MA, Chaturvedi A. A multicenter, retrospective study (RE-ENACT 2) on the use of Razumab ${ }^{\mathrm{TM}}$ (world's first biosimilar ranibizumab) in wet AMD, DME, RVO and myopic CNV. J Clin Exp Ophthalmol. 2019;10(5):6.

16. Narayanan R, Kelkar A, Abbas Z, et al. Sub-optimal gain in vision in retinal vein occlusion due to under-treatment in the real world: results from an open-label prospective study of intravitreal ranibizumab. BMC Ophthalmol. 2021;21(1):33.

17. James DGP, Mitkute D, Porter G, Vayalambrone D. Visual outcomes following intravitreal ranibizumab for diabetic macular edema in a pro re nata protocol from baseline: a real-world experience. Asia Pac J Ophthalmol (Phila). 2019;8(3):200-5. 